\title{
Formation and functions of alter personalities in dissociative identity disorder: a theoretical and clinical elaboration
}

\begin{abstract}
Dissociative identity disorder DID is a chronic complex psychiatric condition related to cumulative psychological traumatization in childhood. It is characterized by a marked disturbance of identity due to the presence of distinct personality states and repetitive dissociative amnesias which interfere with the continuity of the affected person's autobiography. These personality states alter personalities recurrently take control of or influence the individual undermining one's sense of self and agency. Although working with alter personalities is the hallmark of psychotherapy in DID, a detailed and specific clinical and theoretical psychopathology of alter personalities do not exist yet. Hence, the present paper addresses the formation and functions of alter personalities in DID. The hypotheses, proposals, and assumptions developed in this paper have been derived from experiences inintensive treatment of a very large number of patients with DID over more than two decades. The authors propose that the reconciliation between diverse perspectives about one's internal world and external reality carried by various personalities is necessary for successful treatment of DID. The hallmark of dealing with alter and host personalities constitutes of the elimination of misperceptions of them about each other personality state and even about themselves.This requires an analysis of the missions and functions of alter personalities which are usually different than the perceived conceptualizations. This recognition usually increases the therapeutic alliance and even consent between the therapist, and alter and host personalities and decreases the duration of treatment. The present paper is a preliminary one on this subject and may serve as a basis both for further theoretical elaborations as well as for development of hypotheses in empirical research devoted to understanding the operations of human mind when exposed to stress in particular as well as the mechanisms of effective therapeutic interventions in those conditions.
\end{abstract}

Keywords: psychotherapy, trauma, dissociation, alter personality, identity
Volume 6 Issue 6 - 2016

\author{
Erdinc Ozturk,' Vedat Sar ${ }^{2}$ \\ 'Department of Social Sciences, Institute of Forensic Medicine, \\ Istanbul University, Istanbul, Turkey \\ ${ }^{2}$ Department of Psychiatry, Koc University School of Medicine \\ (KUSOM), Istanbul, Turkey
}

\begin{abstract}
Correspondence: Vedat Sar, Department of Psychiatry, Ko University School of Medicine KUSOM, Rumelifeneri Yolu, Sariyer 34450 Istanbul Turkey, Email vsar@ku.edu.tr
\end{abstract}

Received: November 05, 2016 | Published: December 07, 2016

\section{Introduction}

Dissociative identity disorder (DID) is a chronic post-traumatic syndrome related to adverse childhood experiences of cumulative and relational type including childhood abuse and/or neglect. The disorder is clinically characterized by marked disruptions of identity due to the presence of distinct (alternate) personality states which take one's control episodically. ${ }^{1}$ Recurrent dissociative amnesias interfering with autobiographical memory accompany the identity disturbance. The most effective treatment of DID is phase-oriented psychotherapy ${ }^{2}$ which is composed of stabilization (alleviation of daily crises), trauma work (realization of the dissociated aspects of traumatic experiences), and integration (unification of distinct mental structures). While such treatment techniqually covers diverse modalities including almost all generic elements of psychotherapy in general, successful treatment of DID, nevertheless, requires mastery in dealing with alter personalities as a skill of the therapist at least during certain stages of treatment. Textbooks on treatment of DID address the issue of how to deal with this subject. ${ }^{3}$ These descriptions usually underline general rules such as handling alter personalities evenhandedly, establishing contracts with them, correcting their trauma-related cognitive errors, and utilizing fusion rituals. However, the lack of a more specific theory, modeling, and algoritms about alter personalities keeps the treatment of DID as a challenge for average clinician. For instance, as a document written by consensus of several international experts in the field, the ISSTD Guidelines ${ }^{4}$ underlines the importance of working with alter personalities in psychotherapy of DID but it does not cover detailed information about how they can be dealt with in treatment.
Existing definitions of alter personality are based on the assumption that they constitute discrete mental states with their own sense of self and sense of agency. ${ }^{5} \mathrm{Kluft}^{6}$ defined alter personality as "a disaggregated self state" that "is the mental address of the relatively stable and enduring particular pattern of selective mobilization of mental contents and functions, which may be behaviorally enacted with noteworthy role-taking and role-playing dimensions and sensitive to intrapsychic, interpersonal, and environmental stimuli.... It has a sense of its own identity and ideation, and a capacity for initiating thought processes and actions" p.51 Putnam ${ }^{7}$ conceptualized alter personalities as "highly discrete states of consciousness organized around a prevailing affect, sense of self (including body image) with a limited repertoire of behaviors and a set of state dependent memories" (p.103). Host personality is, on the other hand, the personality state which keeps one's control most of the time. Alter personalities may influence or even replace the host personality to transiently make it inactive. While these definitions delienate what an alter personality is, they do not tell much about their ways of formation and their missions and functions. We assume that these aspects are crucial for training a therapist who is expected to treat DID succesfully. In this paper, we try to come up with concrete descriptions of functions and missions of alter personalities and try to provide direct advise about how to approach them. To provide first a background, we begin with a proposal to explain the formation of alter personalities.Our basic assumption is that alter personalities emerge due to an unsuccessful attempt of the person to process a traumatic experience.Hence, we propose that the goal of their emergence is processing the unresolved experience. 


\section{Emergence of alter personalities}

Most authors agree about the relationship of emergence of alternate personalities with developmental traumatization. ${ }^{8}$ One widely accepted understanding is the natural tendency of children to dissociate. In fact, emergence of alter personalities starts in childhood. Simply formulated, the child who is faced with an unbearable event, experiences estrangement: i.e. he or she assumes that the subject being exposed to this traumatic condition was not oneself but someone else. Although serving as a way of coping at the beginning, such estrangement interferes with realization of the experience; i.e. the processes of accomodation and assimilation do not occur and integration of the experience to one's autobiography becomes impossible. ${ }^{9}$ This interrupted process may end up in a clinical condition soon or later; i.e. the subject may develop an overt dissociative disorder in childhood, adolescence, or adulthood while the incomplete trauma process remains dormant. Sar and Ozturk propose that ${ }^{8}$ the clinical syndrome of dissociative disorder becomes manifest after the "traumatic turning point" which does not need to be the worst traumatic experience in the life of the subject.The postponed process may take start in any age; usually becoming clinically manifest in early twenties of age.

According to the proposal of the authors, in order to overcome and process the trauma (immediately or later; i.e. as postponed work), the subject evaluates himself or herself (to a limited extend) and the traumatic experience from diverse perspectives. These perceptions about oneself which originate from diverse perspectives, aggregate around multiple foci. Related emotions, thoughts, and behavior styles are linked to these foci over time. These aggregates become alter personalities following repeated utilization. The host personality can not process trauma after emergence of alter personalities at all. New and past traumatic experiences are dealt by alter personalitieswith. These endevours become even more successful in coping than the operations of the host personality for a while. However, unprocessed traumatic experiences create a negative impact at least on the host personality. This becomes manifest as dissociative depression, dissociative amnesia, and suicidal ideation. ${ }^{10}$ Although the host personality may have suicidal ideation, this intention can not acted by the host. The unprocessed trauma becomes clinically apparent in alter personalities through suicide attempts, affect dysregulation, and self-mutilative behavior.In fact, self-mutilative behavior is an act conducted by alter personalities targeting the host personality.

Each alter personality has independent and different judgements about these internal and external processes because an alter personality is associated with a particular group of perceptions of the individual about oneself that was experienced in the context of a limited aspect of reality. Each alter personality recognizes its own existence and the traumatic experiences in a "single-minded way" rather than utilizing self-reflection: ${ }^{11}$ i.e. just in the direction of its own mission and origin. An alter personality is not fully understood by other alter personalities either, except by those of internal helper type. These conditions increase conflicts and worsens the clinical status. Letting all alter personalities and the host conceive the missions of alter personalities would paradoxically turn alter personalities to a "bridge between realities" rather than being obstacles to integration.

A traumatic situation is characterized by an impasse, because the individual is faced with the so called trauma paradox: bein unable to respond appropriately when needed to do so. ${ }^{12}$ The subject experiences an inflationist proliferation of alternative solutions to this impass in the mind. These solutions are usually based on mental representations of prior inadequate responses to past stressful experiences. One of these options takes priority for the index traumatic experience, though it rarely leads to a solution. Subsequently, the subject continues to attempt to resolve the impasse by keeping these mental representations in the active memory. Remaining options, likewise being stucked in trauma-related past time, is relegated to inactive memory during these repetitions, either partially or totally. These excluded options form the basis of alter personalities which may develop immediately or in the future. Options of solution for recurrent traumatic experiences and repeatedly evaluated cognitions gradually detach from each other. Formed into alter personalities, the subject tries to use the excluded options to solve newly emerging life problems.

In our view, there may be three ways of alter personality formation in the face of traumatic experiences. One is through estrangement. ${ }^{13}$ An attempt to integrate new perceptions about oneself into the existing ones occurs after each traumatic experience. In conditions when one cannot accomodate to the new reality, a "traumatic break"occurs instead which inteferes with proper flow of the process. The subsequent concentrating on the problem increases the departure from reality despite searching for a convergance between diverse perceptions. The subject tries to adopt feelings, thoughts, and behaviors in his mind which are felt to be at a distance from oneself. However, being unsuccesful in this task, one places them in various orbits around oneself according to their proximity imaginatively.

A further way of alter personality formation is the striving for "auto-reparation". ${ }^{8}$ A realistic ("master") record of the event is saved in the inactive memory as a backup after each traumatic experience which one could not cope with.In order to process the experience, the subject continues to work on the imaginative "study" copies in his active memory. Study copies (which are tried to be converted to realistic ones unsuccessfully) gain autonomy in time and create the basis for alter personalities. Master copy is, however, kept in the inactive memory.

The third way of alter personality formation is about activation of non-utilized potentials, abilities, and additional characteristics as interconnected emotions, thoughts, and behaviors of oneself ${ }^{8}$. Various potential abilities constitute the alter personalities after these repetitions. In order to utilize these capacities better, the host personality creates an identical copy of itself. This tendency of the host personality has two aims. The first one is creating mental entities resembling oneself in order to gain strength; i.e. establishing control which has been lost. The second one is trying to utilize the host's own characteristics which the host cannot benefit from. This second aim is the most important factor in the emergence of secondary alter personalities. From this point of view, secondary alter personalities can be perceived as being more real than the host personality. These alter personalities which are created by the host personality cannot carry the capacities of a host personality. However, the imaginary "copies"of self created by the host for the sake of self-reparation as the host is usually non-functional attempt to replace the host Ersatz and, in fact, this becomes a necessity from time to time, as seen in epochal DID ${ }^{14}$ in particular; i.e. those subjects who switch very rarely that longer periods remain in control of a single personality state. In fact, secondary alter personalities may temporarely replace the host personality as a tentative solution, because the host personality cannot function optimally. That means, the host personality cannot control, change or make complete itself on its own, it can only try to do that by producing mental entities resembling herself (“copies").

It is important that the host (but nor the alternate personalities) recognizes the trauma experience as such. On the other hand, it is important that the therapist (but not necessarily the trauma experience) is recognized as such by all alter personalities. Many therapists focus 
on acceptance of the traumatic experience as such by the host. In fact, recognition of traumatic experience by alter personalities are strategically important because alter personalities (except helper personalities) do not enter into communication with host personality. They consider the host a submissive, useless, and shy individual rather than a traumatized one.Hence, the acceptance of traumatic experience as such is important for the change of the misperceptions of alter personalities about host. The host's fear of the traumatic experience becomes an urge for persecutory alter personalities to take revenge.

\section{Four levels of detachment from reality}

Integration involves looking at oneself repeatedly in the context of different versions of reality, trying to perceive oneself in each of these contexts, and making sociopsychological links between these various reality versions as well as between perceptions of self. Thus, the main goal of treatment of a person with DID is becoming oneself which is identical with integration in our view. Kluft ${ }^{15}$ stated once, that DID was a "multiple reality disorder". Alternatively, one may consider dissociation as a way of "reality regulation". ${ }^{16}$ Traditionally, disturbances of reality testing have been considered as belonging to the domain of psychotic disorders. "Trance logic" has been utilized as a concept describing the ability of living in contrasting realities which is one of the hallmarks of dissociation. ${ }^{17}$ Dissociation is, in fact, a striving of the host and alter personalities (an internal system composed of contrasting "realities") to adjust to the absolute external reality. From the perspective of the external world (i.e. a "normal" person in the absolute reality), this appears as a pathological condition. From the internal system's (internal reality composed of alter personalities) perspective,this is a condition aimed at processing the traumatic experience and at adjustment to the external world (absolute reality) despite the contrasting situation in the internal world even though this condition is characterized by daily clinical crises. One consequence of this consideration might be to determine how to benefit from these phenomena in treatment of DID.

In order to inquire the levels of reality, we propose five stages of reality regulation: Utopia, fantasy, imagination, dream, and reality. Utopia is a plan or an idea of an integrated person which is not possible to turn to reality which may be dramatic and even creative. It is a goal to achieve in ideal conditions. Fantasy is an internally cohesive thought content composed of fantastic elements. The force behind fantasy is an unsatisfied and even not yet consciously perceived wish. In dissociative individuals, the internal world composed of fantasies may become more satisfying than the external world of actions and reality. A healthy person has to differentiate fantasy from imagination. The latter is the ability of forming mental images, sensations, and concepts in a moment when they are not perceived through sight, hearing, or other senses. It helps to provide meaning to experiences and a capacity to better understand the obtained knowledge.Imagination facilitates awareness about experiences and the process of obtaining the knowledge which leads to easier utilization of these capacities. Lack of imagination inhibits this process. Hence, the therapist should benefit from the still existing capacity of imagination in cases of DID. Imagination is predominantly a strength of alter personalities although the host can also utilize this to some degree. It is is a fundamental facility through which people make sense of the world, and it also plays a key role in learning process. Imagination can also be expressed through fantasies. last but not least, dream represents wishes, reality represents facts.

Dream, reality, fantasy, and utopia are concepts which are needed both to explain functions of alter personalities and the ways how trauma is processed. The host personality strives to convert his or her utopias to reality unsuccessfully. The subject experiences tremendous disillusion due to this impasse. While, in fact, striving to convert his or her utopias to reality, the subject is compelled to deal with fantasies and imaginations through her alter personalities instead. However, utopia is foreclosed from fantasy and imagination. Converting an utopia to reality is more difficult than converting a fantasy to reality. This is difficult to conduct in a dissociative condition. The host ignores alter personalities because most of the latter are aware of the impossibility of this attempt and oppose to the host in this direction. This is the main reason why the persecutory alter personalities usually criticize the host. On the other hand, the host's utopia is usually the acceptance of its wishes by all alter personalities. The host personality can make its first steps toward turning its fantasies to reality after first fusions with alter personalities. The next step would be renunciating from fantasies but devoting the intention to turning dreams to reality instead. Alter personalities try to convert their fantasies to reality as well. However, the goal of their fantasies is usually limited just to take control for a time period i.e. a few hours.

The therapist is usually faced with depression in the host personality of a dissociative patient. ${ }^{17}$ The depression of the host personality originates from the lack of the experience of unity. The desire to achieve unity remains only a utopia or a fantasy in this stage. The partial awareness of the impossibility of experiencing unity creates a disillusion in the host personality during this period. Psychotherapy is able to help that. Alter personalities; on the other hand, do not have any overt interest in achieving unity. The host personality is anesthetic and numb: i.e., the subject cannot utilize emotions when being in host personality unless entering the world of imagination and fantasy. Thus, early fusion of imaginating and fantasizing alter personalities to the host personality may soften the numbness of the host personality and thereby facilitate psychotherapy.

\section{Functions and missions of alter personalities}

We assume that each alter personality covers five components: a mission, information related to the trauma experience including cognitions, certain emotions, information about the (non-dissociative) past of the person, and determinants for the relationships of the person in a social environment. Alter personalities may have sociological, psychological, or regulating functions which constitute their mission in general. Each of the five components of the host personality and of the persecutory alter personality (as an example of alter personalities) can be described as follows:

\section{Host personality}

i. Compared to alter personalities, the host personality can utilize more multi-focus judgement (i.e. better self-reflection), is more flexible (less self-certain), and is more prone to integration. It has usually a depressive flavor ("dissociative depression") and suffers from intense anxiety.The latter may turn to a major depression and suicidality.

ii. The host personality is partly amnesic to traumatic experiences. Integration of alter personalities to the host usually facilitates reestablishment of a continuous autobiographical memory allowing better chronological flow of the personal history.

iii. The host is carrying emotions of depressive flavor beside being numb in general.

iv. The host remembers personality characteristics of non-dissociated period of life due to dissociative amnesias.

v. The host is sociallly more or less isolated, remins in contact with his or her family or a few friends. 


\section{Persecutory alter personality}

i. It is single-minded in judgement. The persecutory alter personality tries to disconnect the relationship of the host with perpetrators. In fact, it is a helper character at the beginning. However, the revictimization experiences of the host turns it to a persecutor. It carries all defense and coping mechanisms which were utilized furing the traumatic experience.

ii. The persecutory personality remembers traumatic experiences and tries to develop solutions in a rather masculine way. It intends to destroy perpetrators and also the host as it allows perpetrators to victimize the subject.

iii. It carries anger-related emotions.

iv. The persecutor is not interested in the character of the host. It cosniders the host just as a submissive character and ridicules it. The persecutor is self-focused, it repersents the powerful aspect of the pre-dissociated person.

v. The persecutor is attracted by individuals who look like powerful and wants to develop relationship with them (Table 1).

Table I Missions of alter personalities in a case of dissociative identity disorder (23 year old female patient with college education)

\begin{tabular}{|c|c|c|c|}
\hline Personality & Host's Perception & Real Condition & Mission \\
\hline Host 23 year old & $\begin{array}{l}\text { Attempts suicide, feels old, } \\
\text { too much social responsibility, } \\
\text { exposed to high expectancies }\end{array}$ & $\begin{array}{l}\text { Would carry out the expected functions } \\
\text { if not traumatized-dissociated. Helpless- } \\
\text { suicidal }\end{array}$ & $\begin{array}{l}\text { No capacity to achieve expectancies. } \\
\text { The pathological status itself is a call for } \\
\text { integration. }\end{array}$ \\
\hline Yavuz, 14 year old & $\begin{array}{l}\text { Male child, religious, condition } \\
\text { before made "dirty" by a } \\
\text { boyfriend when I } 4 \text { year old. } \\
\text { Wants to be "clean". Keeps the } \\
\text { host away from alcohol. Carries } \\
\text { balance. }\end{array}$ & $\begin{array}{l}\text { Religiosity as coping mechanism against } \\
\text { trauma. }\end{array}$ & $\begin{array}{l}\text { Maintaining mutual adjustment inside of } \\
\text { the personality (internal) system }\end{array}$ \\
\hline Ahmet, 27 year old & $\begin{array}{l}\text { Advises to carry professional } \\
\text { dutiesperfectly and to maintain a } \\
\text { well organized life. }\end{array}$ & $\begin{array}{l}\text { Carrying repetitions as a way of relief. } \\
\text { Interested in order, obsessive. }\end{array}$ & Creating order in the inner system \\
\hline Sadiye, 37 year old & $\begin{array}{l}\text { Persecutory "old"lady, wants to } \\
\text { marry a rich man, wants to do } \\
\text { whatever she wants to. Angry } \\
\text { about host. }\end{array}$ & $\begin{array}{l}\text { Tries to escape from trauma and anxiety. } \\
\text { Fighter,helper, hopeful }\end{array}$ & Pursuing happiness, solitude, pleasure \\
\hline Ayse, 39 year old & $\begin{array}{l}\text { Poet, resembles the patient, as if } \\
\text { coming out from old novels and } \\
\text { times. Shy. }\end{array}$ & $\begin{array}{l}\text { Interested in fantasies and ideals. Helper. } \\
\text { Creative. }\end{array}$ & $\begin{array}{l}\text { Finding creative solutions to conflicts of } \\
\text { the inner system }\end{array}$ \\
\hline Safiye, 23 year old & $\begin{array}{l}\text { Seductive, wants to marry a rich } \\
\text { man (similar toSadiye). Feels like a } \\
\text { highschool pupil,fighter. }\end{array}$ & $\begin{array}{l}\text { Powerful woman, leading alter, has best } \\
\text { solutions, can persuade other alters }\end{array}$ & Seeks novelty, persistent, not submissive \\
\hline Kerem, 10 year old & $\begin{array}{l}\text { Wants to escape from } \\
\text { oppression and to become } \\
\text { herself,cheerful,live, creative, stops } \\
\text { her father and others talking, } \\
\text { curious, close to host. }\end{array}$ & $\begin{array}{l}\text { Male child alter, life-energy, activates the } \\
\text { host, compensates the depressive aspect. } \\
\text { sWants to grow and take control. Behaves as } \\
\text { others did to him. }\end{array}$ & $\begin{array}{l}\text { Close to host and the status before } \\
\text { s trauma }\end{array}$ \\
\hline Seda, 27 year old & $\begin{array}{l}\text { Cheerful, live,interested in movies } \\
\text { and music, positive, lesbian }\end{array}$ & Lives in cheerful circles, hopeful, socialized, & $\begin{array}{l}\text { Compensates the depressive- } \\
\text { introverted host, helper }\end{array}$ \\
\hline Old lady, 80 year old & $\begin{array}{l}\text { Hates the father of the patient, } \\
\text { angry }\end{array}$ & $\begin{array}{l}\text { Protects alters from perpetrators } \\
\text { (inside and outside). In fact, destroys the } \\
\text { relationship between alters and the patient's } \\
\text { father (like the patient's mother does) }\end{array}$ & Wisdom, guidance in general \\
\hline
\end{tabular}

\section{Types of alter personalities}

Several authors have described alter personality types: Child, persecutor, helper, opposite gender, memory trace, and suicidal alter personalities are among them. ${ }^{6}$ In our view, a classification of alter personality types should take into account their functions rather than its mere appearance. One classification may be based on their relationship with narratives: Normalizing, exaggerating, sociological, memory type, confabulating, etc. A second way of classification takes into account the relationship between alter personalityand the "sociological self"18 which is defined as the aspect of the individual devoted to adjustment to the social environment and to protecting one's psychological self (i.e. one's unique aspects): Claiming, polarizing, competing, abusing, distorting, cruel etc. The third way of classification is based on the alter personality's activity in creating confusion for the system. Namely,in front of the stirivings of the host personality to take control, the alter personality interferes with the host with these actions and even leaves it useless. Even in such a situation the host maintanins its link with certain alter personalities; i.e.those who are secret-keeping and/orreality-oriented. The host personality can not maintain contact with reality-distorting, anarchistic, and fantasizing alter personalities because they reject to be in contact with the host.

Persecutory alter personalities are hostile to the host personality.

Persecutory personalities originate from the shame and anger related to the traumatic experience and to the perpetrator. In fact, one of the helper alter personalities turns to a persecutor after having observed that the host personality cannot cope with trauma and is 
revictimized. ${ }^{19}$ This transition has the aim of protecting the internal system and the person as a whole. Although this transition creates a risk for the internal system, the persecutor reacts to threats coming from external world better. The traumatized person cannot reconcile the traumatic experience and the perpetrator. The persecutor blames the host due to its inefficiency in processing the trauma and saving itself from the traumatic experience. This leads the persecutor to stay at a distance to the host which can be diminished after reconciliation. The patient is likely to relate to her host personality and to her social environment through the persecutory alter which covers the most basic and destructive features of the sociological self. In a person with alter personalities, these defenses allow the adjustment to daily life. Without this defenses, an alter personality becomes a submissive one like the host. The persecutory personality serves as a shield against new traumatic experiences. The lack of reconciliation leads to selfmutilative behavior executed under the control of the persecutory alter personalities.

Helper alter personalities display a benevolent attitude toward the host personality. Helper personalities take their origin from the ability of the host personality to look at herself and at the present time from future. This is initially a defensive response of the host personality against persecutory alter personalities. This defensive response becomes a helper alter personality only after repetitive use. Thus, chronologically, it is the persecutory alter personality who creates the first split. Helper alter personalities constitute the second split, and as such, constitute a defense against the first split. These two successive splits constitute the basic dissociation. Further alter personality formations are only of secondary nature. In order to cope with the first split, the host personality produces companions resembling her as a second defense. The alter personalities developing in the second phase originate from the desire of the host personality to regain her previous strength and from her striving to create alter personalities resembling herself who share the same aim with her.

\section{Alter personalities in psychotherapy}

A thoroughly description of the psychotherapy of dissociative disorders and all types of alter personalities exceeds the limits of this paper. However, providing a very short introduction is indicated here because the theoretical elaborations in this paper have several concrete implications for psychotherapy of DID. While alter personalities constitute only one dimension of psychopathology in DID, they are considered as key elements in thepsychotherapy of the disorder. ${ }^{20}$ Alter personalities determine the appropriate psychotherapeutic approach and timing of interventions: i.e., what and when should be the focus of inquiry. Number and diversity of alter personalities determine the suitable approach to the host personality. Rather than the total number of alter personalities, the severity of the clinical condition depends on the issue of how many times an alter personality has gone through a psychological processing. Alter personalities determine the resistances in psychotherapy and the overall attitude (i.e.therapeutic mutuality) of thepatient toward the therapist. This also determines the treatment algorithm.

Successful integration of an alter personality depends on recognition of its mission as a way of solution to by the person herself. Both from the perspectives of host and alter personalities, knowing the missions of the host and alters diminishes phobic avoidance of each other. This diminishes misperceptions between them.Each alter personality with a psychological mission contributes to the activation of the patient when fused to the host personality. This can be accomplished only after identification of the missions of the alter personality. After integration of the alter personalities with a psychological mission, the alter personalities with a regulating mission should be fused as the second step. Alter personalities with a sociological mission represent the past and the present; they are to be fused at the end stage of integration in treatment. The origin of resistance to treatment ${ }^{21}$ usually originate from misperception or lack of information of the host about alter personalities' aims and functions. One of the aims of psychotherapy is to inform the host about alter personalities' mission and functions. As a second step, the mission and functions of host and other alter personalities to each alter personality. In this way, conflicts, avoidance, struggle, and lack of adjustment between host and alter personalities as well as between various alter personalities themselves would be solved. This approach would make integration more acceptable for each of them, the required treatment period would be shortened and crisis situations would be less frequent.

Knowing why there is a need for a particular alter personality in a given moment, and knowing its function and missions help the therapist to determine the severity of thedissociative disorder. Although the types of host and alter personalities are similar in all cases of DID, their number and diversity is correlated with the severity od the condition. For example, the frequent dominance of persecutory alter personalities points to the distance between host and alter personalities. Such distance predicts suicide attempts and selfmutilative behavior. In condition that the disorder is in its beginning period, helper alter personalities take the control predominantly which would lead to less severe clinical condition.In DID, from the perspective of alter personalities, flashbacks and trauma-related hallucinations are experienced. This may take the form of a brief psychosis. In host personality, experiences of flashbacks is more common; such a condition is close to PTSD.Psychotic and trauma-related hallucinations occurduring contact with an other alter personality, for example, if two alter personalities take to control simultaneously. Persecutor alter personality may show psychotic hallucinations to the host to frighten and re-traumatize him. Trauma-related hallucinations (e.g. a parted or hanged body etc.) can be experienced by any alter personality who witnessed a traumatic experience. Such a situation dissolves if the missions and functions of an alter alter personality are identified by both host and alter personalities.

\section{Conclusion}

The assumptions and hypotheses presented in this paper have been derived from experiences on treatment of a very large group of clients with chronic dissociative disorders gathered over more than two decades. The lack of a specific drug treatment for and the rewarding outcome of psychotherapy in dissociative disorders make these clinical phenomena an avenune for innovation in psychiatry. However, such innovation requires a coupling of research with appropriate modeling which has to go beyondany "linear" thinking on causality. Hence, the present paper is an attempt to broaden the thinking on algoritms of emergence and psychotherapy of dissociative disorders as specified in an elaboration on psychopathology of alter personalities; i.e. the hallmark of DID. Neverrheless, this avenue is promising for further illumination of the operations of human mind when its capacity of adaptation and survival are challanged by unexpected deviations of external reality.

\section{Acknowledgments}

None.

\section{Conflicts of interest}

Author declares there are no conflicts of interest. 


\section{Funding}

None.

\section{References}

1. American Psychiatric Association. Diagnostic and statistical manual of mental disorders, Ed.5. Washington DC: American Psychiatric Association. 2013.

2. Steele K, Van der Hart O, Nijenhuis ERS. Phase-oriented treatment of structural dissociation in complex traumatization:overcoming traumarelated phobias. J Trauma Dissociation. 2005;6(3):11-53.

3. Kluft RP. An overview of the psychotherapy of dissociative identity disorder. Am J Psychother. 1999;53(3):289-319.

4. Chu JA, Dell PF, Van der Hart O, et al. International Society for the Study of Trauma and Dissociation Guidelines for Treating Dissociative Identity Disorder in Adults (3rd edn). J Trauma Dissoc. 2011;12:115-187.

5. Spiegel D, Lewis-Fernandez R, Loewenstein R, et al. Dissociative disorders in DSM-5. Depression \& Anxiety. 2011;28(9):824-852.

6. Kluft RP. The phenomenology and treatment of extremely complex multiple personality disorder. Dissociation. 1988;1(4):47-58.

7. Putnam FW. Diagnosis and treatment of multiple personality disorder Guilford Press, USA. 1989. p.1-315.

8. Sar V, Öztürk E. What is trauma and dissociation? J Trauma Practice. 2005;4(1-2):7-20.

9. Horowitz MJ. Stress response syndromes. Hosp Community Psychiatry. $1986 ; 37(3): 241-249$.

10. Şar V, Akyüz G, Öztürk E, et al. Dissociative depression among women in the community. J Trauma Dissociation. 2013;14 (4):423-438.

11. Şar V, Öztürk E, Islam S, et al. Zwischen Selbstreflexion und SelbstÜberszeugtsein: Kognitive Einsicht bei dissoziativen und schizophrenen Störungen und das Dissoziationsparadox. Zeit heilt nicht alle Wunden: Kompendium zur Psychotraumatologie. In: Özkan I \& Sachsse U (Eds.), Vandenhoeck Ruprecht, UK. 2012. p.1-172.
12. Fischer G, Riedesser P. Lehrbuch der Psychotraumatologie. Ernst Reinhardt Verlag, UK. 1999.

13. Şar V, Alioğlu F, Akyüz G. Depersonalization and de-realization in selfreport and clinical interview: the spectrum of borderline personality disorder, dissociative disorders, and healthy controls. J Trauma Dissociation. 2016;17:1-17.

14. Kluft RP. Clinical presentations of multiple personality disorder. Psychiat Clin North Am. 1991;14(3):605-609.

15. Kluft RP. Basic principles in conducting the psychotherapy of multiple personality disorder. In: RP Kluft \& CG Fine (Eds.), Clinical perspectives on multiple personality disorder, American Psychiatric Press, USA. 1993. p.1-50.

16. Şar V, Öztürk E. Psychotic presentations of dissociative identity disorder. In: PF Dell \& JO Neil (Eds.), Dissociation and the dissociative disorders: DSM-V and beyond, Routledge Press, USA. 2009. p.1-12.

17. Şar V. Dissociative depression is resistant to treatment-as-usual. $J$ Psychology Clin Psychiatry. 2015;3(2):000128.

18. Şar V, Öztürk E. Functional dissociation of the self: a sociocognitive approach to trauma and dissociation. $J$ Trauma Dissociation. 2007;8(4):69-89.

19. Ross CA. Dissociative identity disorder. Diagnosis, clinical features, and treatment of multiple personality (2nd edn). John Wiley, USA. 1997. p. $1-452$.

20. Kluft RP. Dealing with alters: a pragmatic clinical perspective. Psychiat Clin North Am. 2006;29(1):229- 242.

21. Kluft RP. The difficult-to-treat patient with dissociative disorder. In: En M. J. Dewan, \& R.W. Pies (Eds.), The Difficult-To-Treat Psychiatric Patient, American Psychiatric Publishing, USA. 2001. p.1-242. 